The Astrophysical Journal, In PRESS, March 22, 2021

Typeset using LATEX twocolumn style in AASTeX63

\title{
Inward Propagating Plasma Parcels in the Solar Corona: Models with Aerodynamic Drag, Ablation, and Snowplow Accretion
}

\author{
Steven R. Cranmer, ${ }^{1}$ Craig E. DeForest,${ }^{2}$ and Sarah E. Gibson ${ }^{3}$ \\ ${ }^{1}$ Department of Astrophysical and Planetary Sciences, Laboratory for Atmospheric and Space Physics, University of Colorado, Boulder, CO 80309, USA \\ ${ }^{2}$ Southwest Research Institute, 1050 Walnut Street, Suite 300, Boulder, CO 80302, USA \\ ${ }^{3}$ National Center for Atmospheric Research, 3080 Center Green Drive, Boulder, CO 80301, USA
}

\begin{abstract}
Although the solar wind flows primarily outward from the Sun to interplanetary space, there are times when small-scale plasma inflows are observed. Inward-propagating density fluctuations in polar coronal holes were detected by the COR2 coronagraph on board the STEREO-A spacecraft at heliocentric distances of 7 to 12 solar radii, and these fluctuations appear to undergo substantial deceleration as they move closer to the Sun. Models of linear magnetohydrodynamic waves have not been able to explain these deceleration patterns, so they have been interpreted more recently as jets from coronal sites of magnetic reconnection. In this paper, we develop a range of dynamical models of discrete plasma parcels with the goal of better understanding the observed deceleration trend. We found that parcels with a constant mass do not behave like the observed flows, and neither do parcels undergoing ablative mass loss. However, parcels that accrete mass in a snowplow-like fashion can become decelerated as observed. We also extrapolated OMNI in situ data down to the so-called Alfvén surface and found that the initial launch-point for the observed parcels may often be above this critical radius. In other words, in order for the parcels to flow back down to the Sun, their initial speeds are probably somewhat nonlinear (i.e., supra-Alfvénic) and thus the parcels may be associated with structures such as shocks, jets, or shear instabilities.
\end{abstract}

Keywords: Astrophysical fluid dynamics (101) - Magnetohydrodynamics (1964) - Solar corona (1483) - Solar magnetic reconnection (1504) - Solar wind (1534) - Space plasmas (1544)

\section{INTRODUCTION}

The Sun continuously releases a fraction of its own mass in the form of an accelerating outflow of ionized gas called the solar wind. There are many direct and indirect measurements of the outward propagation of plasma parcels above the Sun's surface (see, e.g., Sheeley et al. 1997; Schwenn 2006; Kohl et al. 2006; Tokumaru et al. 2010; Abbo et al. 2016). However, we do sometimes observe plasma flows that go back down to the solar surface. Close to the Sun, there are supra-arcade downflows in hot active regions (McKenzie 2000; Savage et al. 2012), infalling droplets within cool prominences (Haerendel \& Berger 2011), and "coronal rain" occurring in loop-like structures (Müller et al. 2005; Antolin et al. 2015). Further up, at heliocentric distances of about $2-6 R_{\odot}$ (solar radii), there are signatures of inflowing parcels in visible-light coronagraphy (Wang et al. 1999; Wang \& Sheeley 2002; Sheeley \& Wang 2014; Dolei et al. 2014; Sanchez-Diaz et al. 2017). In-

Corresponding author: Steven R. Cranmer flows at even larger distances of 7-15 $R_{\odot}$ were detected by DeForest et al. (2014) via the careful Fourier filtering of coronagraphic data from the COR2 instrument on the STEREO-A spacecraft (Howard et al. 2008).

It is likely that the reason no coronal inflows are observed above $15-20 R_{\odot}$ is because this is the vicinity of the Sun's Alfvén radius $r_{\mathrm{A}}$. This boundary-sometimes also called the Alfvén surface or the Alfvén point- is where the solar wind speed begins to exceed the characteristic wave speeds of all magnetohydrodynamic (MHD) waves. Thus, it acts as a critical surface beyond which information cannot propagate back down to the Sun in the form of linear waves (Weber \& Davis 1967; Belcher \& MacGregor 1976). It is also thought to be an effective "source surface" for heliospheric magnetic flux, since field lines that extend beyond $r_{\mathrm{A}}$ appear to have no way of connecting back down to the photosphere. MHD simulations of the corona-heliosphere system tend to place the Alfvén radius at distances between 10 and $30 R_{\odot}$ (e.g., Pinto et al. 2011; Cohen 2015; Chhiber et al. 2019), and the three-dimensional shape of the surface is often far from spherical. 
The COR2 coronal-hole measurements of DeForest et al. (2014) indicated an overall deceleration for inflowing parcels: from speeds of order $80 \mathrm{~km} \mathrm{~s}^{-1}$ at $12 R_{\odot}$ to speeds of $20 \mathrm{~km} \mathrm{~s}^{-1}$ by the time they reach $7 R_{\odot}$. However, models of MHD-wave propagation (e.g., Tenerani et al. 2016) were not able to reproduce this observed trend. Alfvén or fast-mode waves that propagate radially back to the Sun ought to undergo acceleration, not deceleration. Obliquely propagating MHD waves may decelerate as they become refracted, but they would exhibit the opposite "concavity" (in a radius-versus-speed diagram) than the COR2 data. ${ }^{1}$ Thus, Tenerani et al. (2016) concluded that these parcels are not wavelike oscillations, and may instead be jets from coronal sites of magnetic reconnection. In general, such parcels could be bursty flows associated with localized reconnection events at the tips of streamers or pseudostreamers (Wang et al. 1998; Rappazzo et al. 2012; Sanchez-Diaz et al. 2019), tearing-mode islands from more distributed sites of turbulent reconnection (Simnett 2004; Gosling 2007; Réville et al. 2020), or possibly Kelvin-Helmholtz vortices driven by sheared solar-wind flows (Roberts et al. 1992; Ofman \& Thompson 2011).

This paper explores a range of dynamical models of parcel motion through the corona, and we attempt to identify the model parameters that best reproduce the observed deceleration trend of DeForest et al. (2014). In Section 2 we describe a set of equations of motion inspired by drag-based studies of coronal mass ejections (CMEs). Section 3 generalizes the model to allow for parcels to accrete mass from the ambient solar wind plasma, and Section 4 includes the effects of ablative mass loss from the parcels. Although there are some astrophysical environments where mass gain and mass loss can compete with one another (e.g., cold clouds in the circumgalactic medium; see Gronke \& Oh 2018; Li et al. 2020), here we keep the two models separate and distinct. In Section 5 we describe observational constraints on the initial conditions of the parcel at $12 R_{\odot}$, and we use OMNI in situ data to estimate probability distributions for characteristic inflow speeds at this radius. Notably, we discuss the possibility that downward flows can exist above the Alfvén surface if the initial conditions are nonlinear (i.e., locally supra-Alfvénic). Lastly, Section 6 concludes by summarizing our results, suggesting future improvements to these kinds of models, and discussing some broader implications.

\section{THE AERODYNAMIC DRAG MODEL}

The kinematic interaction between the proposed parcels and the background solar-wind plasma is described using a drag-based formalism adapted from CME modeling (Section

\footnotetext{
${ }^{1}$ Illustrations of this concavity discrepancy can be found in Figure 3, below, or in Figures 2-3 of Tenerani et al. (2016).
}

2.1), with both analytic (Section 2.2) and numerical (Section 2.3) solutions explored below. In reality, the physical origin of the parcel-e.g., magnetic reconnection, shock steepening, or MHD instability-will likely have something to do with its subsequent dynamics. However, here we take a slightly agnostic approach and consider general forces that can apply in many possible scenarios.

\subsection{Governing Equations}

The complete equation of motion for a small coronal parcel would require the inclusion of forces from gravity, the gas-pressure gradient, the wave-pressure gradient (Bretherton \& Garrett 1968; Belcher 1971), magnetic mirror effects (if the velocity distribution is anisotropic with respect to the magnetic field), MHD Lorentz-force terms, and particle collisions. For both the parcel and the surrounding solar wind, many of these forces are expected to be comparable to one another in magnitude. Thus, as in studies of CME kinematics (e.g., Cargill 2004), we will account only for relative differences in forcing between the parcel and its surroundings. By specifying the acceleration of the ambient solar wind as a kind of attractor for the parcel flow, we can neglect the forces that maintain it in its assumed steady-state.

Hydrodynamic and MHD forces of interaction between the parcel and the surrounding solar wind are often referred to as drag forces. When studying the motions of CMEs, there has been some debate about whether this force scales linearly with the relative velocity between the two fluids (as in high-viscosity Stokes drag; see Vršnak 2001), or whether it scales quadratically (as in low-viscosity turbulent drag; see Borgazzi et al. 2009). Comparison with MHD simulations tends to favor quadratic drag (e.g., Cargill et al. 1996; Cargill 2004), so that formalism is used here. Consider a finite parcel with mass $M_{i}$, positioned at a heliocentric distance $r_{i}$ and flowing radially with velocity $u_{i}$. Its equations of motion are

$$
\begin{gathered}
\frac{d r_{i}}{d t}=u_{i} \\
\left(M_{i}+M_{\text {add }}\right) \frac{d u_{i}}{d t}=-\rho_{e} A C_{\mathrm{D}}\left(u_{i}-u_{e}\right)\left|u_{i}-u_{e}\right|
\end{gathered}
$$

where subscript $i$ refers to the internal properties of the parcel and subscript $e$ refers to the external properties of the surrounding corona (see also Chen 1989, 1996; Subramanian et al. 2012; Vršnak et al. 2013; Dolei et al. 2014; Dumbović et al. 2018; Kay et al. 2020). Thus, $u_{e}$ and $\rho_{e}$ are specified as the speed and mass density of the ambient, time-steady solar wind. From the form of Equation (2), it is clear that the asymptotic solution is for $u_{i}$ to approach $u_{e}$ in the limit of $t \rightarrow \infty$.

Figure 1 illustrates a simplified parcel geometry, in which the volume is given by the product of a radial length-scale $L$ and a cross-sectional area $A$. The mass interior to the parcel 


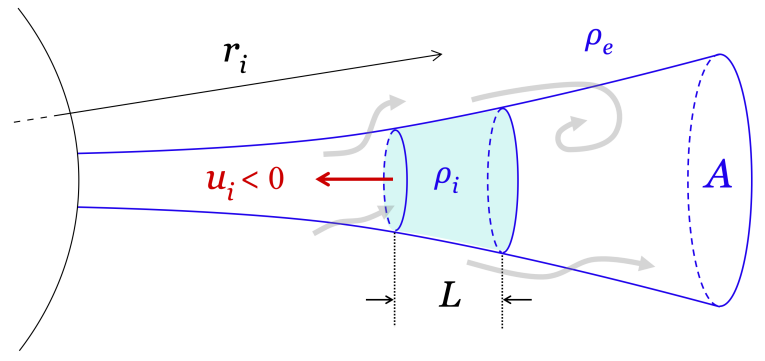

Figure 1. Simplified illustration of a solar-wind plasma parcel moving radially along a superradially expanding magnetic flux tube. See text for definitions of the parcel parameters. Light gray arrows indicate possible streamlines of the surrounding flow (relative to the parcel).

is written as

$$
M_{i}=\rho_{i} A L
$$

where all three components_-including the parcel's mean mass density $\rho_{i}$-can be allowed to vary with radial distance. For the models discussed in this section, it is assumed that $M_{i}$ remains constant in time. Note that the left-hand side of Equation (2) also contains an added mass (or virtual mass) term

$$
M_{\mathrm{add}}=C_{\mathrm{A}} M_{e}
$$

where $M_{e}=\rho_{e} A L$ is the mass of adjacent solar-wind plasma with the same volume as the moving parcel. The dimensionless coefficient $C_{\mathrm{A}}$ is often assumed to be equal to $1 / 2$, and it is discussed in more detail below. The $M_{\text {add }}$ term accounts for the fact that a finite-sized parcel induces motions in the surrounding plasma, and thus carries along a bit of extra inertia. For additional applications of this concept to solar flux tubes, see Spruit (1981), Ryutova \& Priest (1993), and Cranmer \& van Ballegooijen (2005).

Much of the physics describing momentum transfer between the parcel and its surroundings is encapsulated in the dimensionless drag coefficient $C_{\mathrm{D}}$. Computational and observational studies of CMEs have tried to use the large-scale motions of flux ropes to determine likely values for $C_{\mathrm{D}}$ in the corona and inner heliosphere. For example, Cargill et al. (1996) found that varying the magnetic-field geometry in MHD simulations caused $C_{\mathrm{D}}$ to vary between values of 0 and 3 . In this paper, where parcels are tracked primarily between about 7 and $12 R_{\odot}$ (i.e., only over a relatively small dynamic range in distance), $C_{\mathrm{D}}$ is treated as an arbitrary constant. In situations where the parcel is interpreted as a compact MHD plasmoid, an alternate approach could be to take account of the full set of diamagnetic forces, including a "melonseed" effect that accelerates the object in the direction of the weaker external field (see, e.g., Schlüter 1957; Parker 1957; Pneuman \& Cargill 1985; Mullan 1990; Lin et al. 2008).

Realistic values for the added-mass coefficient $C_{\mathrm{A}}$ can be obtained from hydrodynamics. The standard value of
$1 / 2$, used in many drag-based CME models, is appropriate for a spherical object embedded in an incompresible fluid. However, the parcels considered here may become highly anisotropic; i.e., either prolate (longer in the radial direction than in the transverse direction) or oblate (shorter in the radial direction than in the transverse direction). Thus, to take one step beyond the assumption of sphericity, we utilize results for spheroidal objects in incompressible flows (e.g., Lamb 1932; Morel 2015; Fitzpatrick 2017). For a spheroid moving along the direction of its primary axis of symmetry, the total length along its trajectory is specified as $L_{\|}$, and its transverse diameter (i.e., the diameter of the circle one would see by looking down its axis) is $L_{\perp}$. Defining the aspect ratio $\alpha=L_{\|} / L_{\perp}$, the spheroid is oblate if $\alpha<1$ and prolate if $\alpha>1$.

Figure 2 shows the result of an ideal hydrodynamics calculation for $C_{\mathrm{A}}$ as a function of $\alpha$. The exact functions are lengthy, but are given in full in Equations 7.139-7.140 and 7.162-7.163 of Fitzpatrick (2017). In the limit of $\alpha \ll 1$, the analytic result reduces to $C_{\mathrm{A}} \approx 2 /(\pi \alpha)$. Note that $C_{\mathrm{A}} \rightarrow \infty$ in this case because the parcel becomes equivalent to a flat disk whose volume approaches zero. The added mass $M_{\text {add }}$ remains finite and is given by $2 \rho_{e} / \pi$ times the volume of an equivalent sphere that circumscribes the disk exactly. In the limit of $\alpha \gg 1$, the analytic result reduces to $C_{\mathrm{A}} \approx \ln \left(4 \alpha^{2}\right) /\left(2 \alpha^{2}\right)$, which approaches zero as $\alpha \rightarrow \infty$. For the parameters shown in Figure 2, the following fitting formula always agrees with the exact calculation to within $5 \%$,

$$
C_{\mathrm{A}} \approx \frac{2}{\pi \alpha\left(1+0.287105 \alpha^{0.843474}\right)},
$$

and this expression is used in the numerical code that solves for the parcel's evolution, with $L=L_{\|}$and $A=\pi L_{\perp}^{2} / 4$.

In order to determine all parameters in the model, there still needs to be a specification of the parcel's time-dependent shape and anisotropy. The cross-sectional area $A$ is assumed to be identical to that of the large-scale background magnetic field; i.e., we assume that the parcel expands or contracts laterally to fill its local magnetic flux tube. As long as the radial magnetic-field strength $B_{r}$ is known, the principle of magnetic flux conservation provides the relative radial variation of $A \propto B_{r}^{-1}$. Thus, a given model of the solar wind has unique values for $u_{e}(r), \rho_{e}(r)$, and $A(r)$. The evolution in radial length of the parcel is specified with a scaling exponent $\sigma$, as

$$
L=L_{0}\left(\frac{A}{A_{0}}\right)^{\sigma}
$$

where quantities with subscript 0 are the initial conditions at $t=0$. The exponent $\sigma$ will be varied as a free parameter, and it is useful to note that there are several natural values that may pertain in different circumstances: 


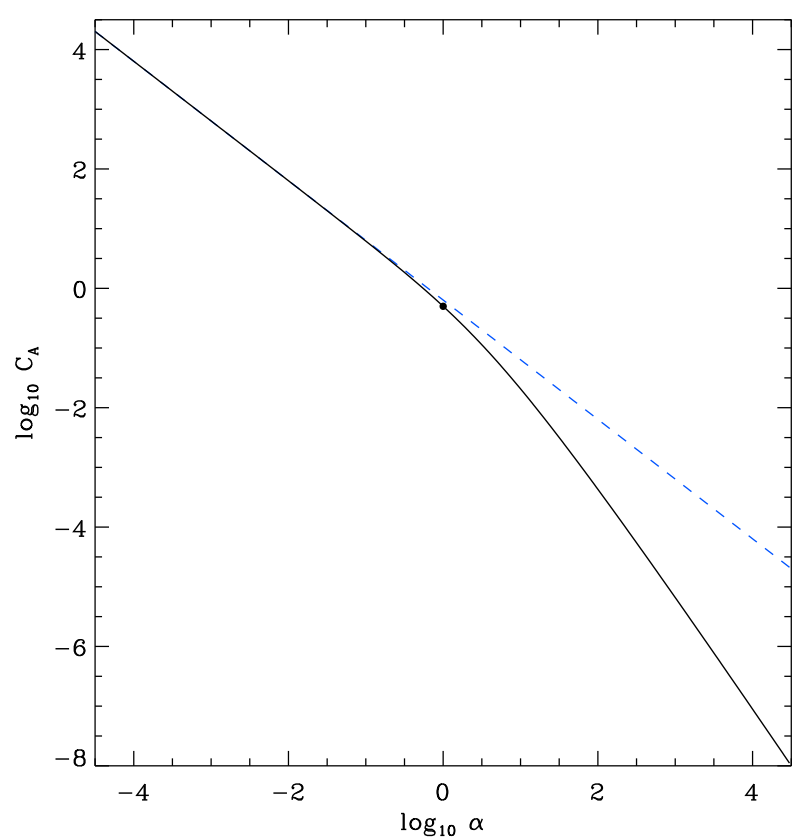

Figure 2. Added mass coefficient $C_{\mathrm{A}}$ plotted versus spheroidal aspect ratio $\alpha$ (solid black curve), computed from formulas given by Fitzpatrick (2017). Also shown is the $\alpha \ll 1$ limit (dashed blue curve) and the spherical case of $\alpha=1$ and $C_{\mathrm{A}}=1 / 2$ (black circle). If the fitting formula given in Equation (5) were overplotted, it would not be distinguishable from the exact solution.

1. A value of $\sigma=-1$ would be appropriate if the volume of the parcel remains constant over time. This may occur for a parcel that retains a constant mass $\left(M_{i}=\right.$ constant) and is close to incompressible ( $\rho_{i}=$ constant).

2. A value of $\sigma=0$ constrains the length $L$ to remain constant. This may be the case if the forces on the front and back faces of the parcel always remain roughly equal to one another. It may also be occurring in the outer corona for observed visible-light "flocculations" that appear to preserve their radial length-scale from about 30 to $90 R_{\odot}$ (see Figure 9 of DeForest et al. 2016).

3. The specific value $\sigma \approx 0.14$ would occur if the parcel's mass is conserved $\left(M_{i}=\right.$ constant) and its internal density remains proportional to the surrounding solar-wind density $\left(\rho_{i} \propto \rho_{e}\right)$. This value comes from the ZEPHYR coronal-hole model discussed below, in which $\rho_{e} \propto r^{-2.45}$ and $A \propto r^{2.15}$ over the relevant range of heights to be compared with the observational data $\left(7-12 R_{\odot}\right)$.

4. Lastly, the value $\sigma=0.5$ would occur if the aspect ratio $\alpha$ of the parcel remains constant over time. An initially spherical parcel may remain so if the lo- cal pressure-gradient forces on it provide a roughly isotropic confinement.

It turns out that the models that successfully explain the observed COR2 parcel deceleration do not depend sensitively on the exponent $\sigma$. Thus, treating it as a free parameter probably does not limit the usefulness of these models. For another approach to simulating the evolution of parcel prolateness in the accelerating solar wind, see Pneuman \& Cargill (1985).

For a specified background solar wind, the above model has two free parameters $\left(C_{\mathrm{D}}\right.$ and $\sigma$ ) and five initial conditions $\left(r_{i, 0}, u_{i, 0}, M_{i, 0}, L_{0}\right.$, and $\left.A_{0}\right)$. The properties that are specified explicitly are $r_{i, 0}, u_{i, 0}, L_{0}$, and the initial value of the density ratio $\rho_{i} / \rho_{e}$. The initial parcel shape is assumed to be spherical, with $A_{0}=\pi L_{0}^{2} / 4$, and $M_{i, 0}$ is computed from the other quantities. For now, the parcel mass is presumed to remain constant over time, with $M_{i}(t)=M_{i, 0}$.

\subsection{Approximate Analytic Solutions}

Equations (1) and (2) will eventually be solved numerically, but it is also helpful to explore closed-form solutions when possible. Thus, we make the simplifying assumption that the wind speed $u_{e}$, background density $\rho_{e}$, and crosssectional area $A$ are all constants as a function of radial distance. This also constrains $\rho_{i}$ and $L$ to be constants as well. The assumption of spherical symmetry for the parcel gives the standard hydrodynamic value for the added-mass coefficient, $C_{\mathrm{A}}=1 / 2$ (Lamb 1932).

Because essentially nothing in the simplified system depends on radial distance (other than the primary dependent variable $u_{i}$ ), Equation (2) can be solved first for $u_{i}(t)$, then the radial trajectory $r_{i}(t)$ can be determined subsequently. It is convenient to define two new dependent variables

$$
U=\left|u_{i}-u_{e}\right| \quad \text { and } \quad Q=\frac{\rho_{i}}{\rho_{e}}+\frac{1}{2},
$$

where the two terms in $Q$ correspond to the $M_{i}$ and $M_{\text {add }}$ terms in Equation (2). For now, the assumption is that $Q$ remains fixed at its initial value $Q_{0}$. Equation (2) is thus rewritten as

$$
\frac{d U}{d t}=-\frac{C_{\mathrm{D}} U^{2}}{Q_{0} L} .
$$

If all quantities on the right-hand side are positive, then $U$ will get smaller as time increases-i.e., asymptotically approaching zero as the parcel's motion becomes entrained into the surrounding solar wind. However, if the initial condition obeys $u_{i}<u_{e}$, then the time evolution of $u_{i}$ can go from negative (inward flow) to positive (entrained outward flow). For a known initial condition $U_{0}$, this equation has an explicit solution

$$
U(t)=U_{0}\left(1+\omega_{0} t\right)^{-1}
$$




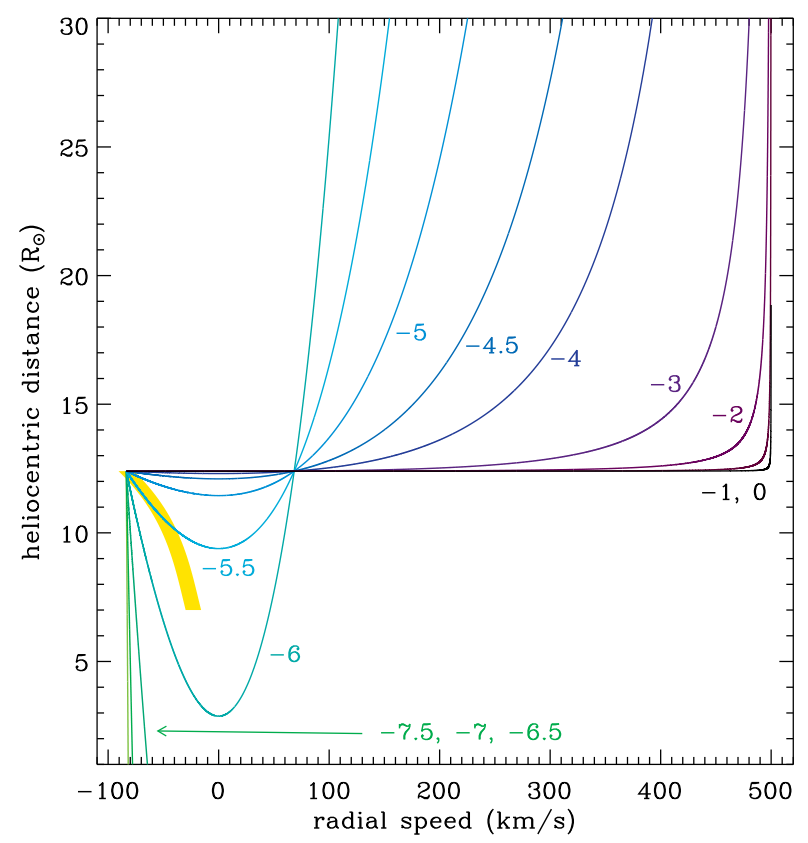

Figure 3. Example analytic solutions for a parcel's radial distance $r_{i}$ versus its instantaneous radial velocity $u_{i}$, modeled with no mass gain or loss (i.e., $M_{i}=$ constant). Solid curves are labeled by their values of $\log _{10} \omega_{0}$, where $\omega_{0}$ is expressed in $\mathrm{s}^{-1}$. Also shown (yellow region) is the observed COR2 inflow ridge from DeForest et al. (2014).

with

$$
\omega_{0}=\frac{C_{\mathrm{D}} U_{0}}{Q_{0} L} .
$$

Note that significant deceleration occurs with a characteristic time scale of $t \sim 1 / \omega_{0}$. The radial position of the parcel is given by integrating Equation (1),

$$
r_{i}(t)=r_{i, 0}+u_{e} t-\frac{U_{0}}{\omega_{0}} \ln \left(1+\omega_{0} t\right)
$$

where a sign convention was adopted that assumes $u_{i}<u_{e}$ for all $t \geq 0$.

Figure 3 shows a range of illustrative solutions of Equations (9) and (11). The initial conditions were chosen to agree with the observations of DeForest et al. (2014) for the largest height at which inflowing parcels were detected: $r_{i, 0}=12.4 R_{\odot}$ and $u_{i, 0}=-83.6 \mathrm{~km} \mathrm{~s}^{-1}$. Here, we also chose a fast solar wind speed of $u_{e}=500 \mathrm{~km} \mathrm{~s}^{-1}$, typical for coronal holes at this distance.

The only other parameter required to implement the above solutions is $\omega_{0}$, and we can estimate likely values by examining the components of Equation (10). From the CME literature, a realistic range of values for $C_{\mathrm{D}}$ seems to be $0.001-1$. The modified overdensity ratio $Q_{0}$ may be as small as 0.5 (if $\rho_{i}<\rho_{e}$ ) and possibly as high as 100 . The observations of DeForest et al. (2014) found that the parcel length-scale $L$ is probably no larger than about $0.5 R_{\odot}$. Although it is unclear what the smallest value of $L$ could be, the fact that these features are observable with STEREO/COR2 probably means they are not much smaller than one pixel in size. Thus, we adopt a single-pixel lower limit for $L$ of about $15^{\prime \prime}$, or $0.016 R_{\odot}$ (Howard et al. 2008). Combining the ranges for $C_{\mathrm{D}}, Q_{0}$, and $L$ with the initial speeds discussed above (i.e., $U_{0}=583.6 \mathrm{~km} \mathrm{~s}^{-1}$ ) gives a possible spread of about eight orders of magnitude for $\omega_{0}$. Figure 3 shows the dynamical solutions for values between $3 \times 10^{-8} \mathrm{~s}^{-1}$ (green curve) and $1 \mathrm{~s}^{-1}$ (black curve).

The green curves in Figure 3 correspond to very low values of $\omega_{0}$. In this case, the drag force on the parcels is extremely weak; they just continue to flow at their initial downward velocity and eventually reach the solar surface. The violet and black curves in Figure 3 correspond to extremely large values of $\omega_{0}$. In that case, the parcels become entrained very quickly into the ambient solar wind and flow out with speeds approaching $u_{e}=500 \mathrm{~km} \mathrm{~s}^{-1}$. Note that all of the entrained solutions pass through the the initial radius $r_{i, 0}$ for a second time at the same velocity, $u_{i}=68.4 \mathrm{~km} \mathrm{~s}^{-1}$. It is possible to write an analytic solution for this velocity, but it requires the use of the Lambert $W$ function (Corless et al. 1996), with

$$
u_{i}=u_{e}\left\{1+\left[W_{-1}\left(-\frac{u_{e}}{U_{0}} e^{-u_{e} / U_{0}}\right)\right]^{-1}\right\},
$$

and these solutions all exhibit $\omega_{0} t=0.35207$.

Figure 3 also shows a fit to the "inflow ridge" (yellow region) that indicates the observed trend of parcel deceleration (DeForest et al. 2014) and is given by

$$
u_{i, \mathrm{fit}}=118.39-46.739 r_{i}+5.5295 r_{i}^{2}-0.24790 r_{i}^{3}
$$

where $r_{i}$ is in units of $R_{\odot}$ and $u_{i \text {,fit }}$ is in units of $\mathrm{km} \mathrm{s}^{-1}$. This fitting formula is valid only between 7 and $12.4 R_{\odot}$ and should not be applied outside this range. Note, however, that no choice of $\omega_{0}$ matches the data well. Like the linear MHD-wave modes studied by Tenerani et al. (2016), the model curves have the opposite concavity as the observed trend.

\subsection{Numerical Solutions}

It is possible that the analytic model described in Section 2.2 fails to match the data because its assumptions about constant values for the model parameters (e.g., $u_{e}, \rho_{e}, A, L$ ) were too simplistic. Thus, more realistic radial dependences for these plasma properties can be adopted, and the original drag equations can be solved numerically. Figure 4 shows coronal-hole parameters from ZEPHYR, a one-fluid model of turbulent heating and acceleration along open magnetic flux tubes (Cranmer et al. 2007). Along with $u_{e}(r), A(r)$, and $\rho_{e}(r)$, we also show the radial Alfvén speed

$$
V_{\mathrm{A} r}=\frac{B_{r}}{\sqrt{4 \pi \rho_{e}}}
$$




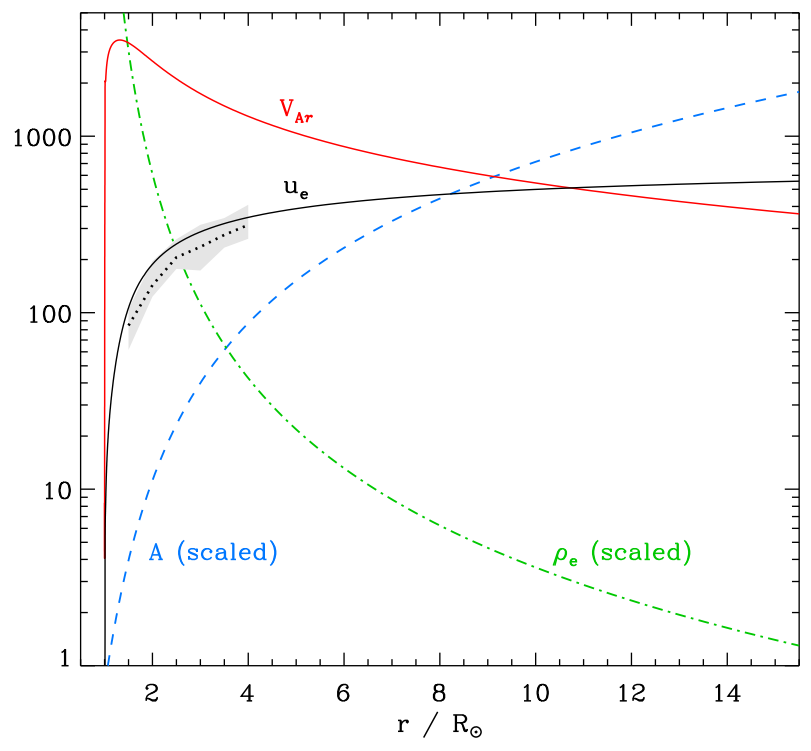

Figure 4. Radial variation of several coronal-hole parameters from the ZEPHYR model, including the wind speed $u_{e}$ (black solid curve) and Alfvén speed $V_{\mathrm{A} r}$ (red solid curve), both given in units of $\mathrm{km} \mathrm{s}^{-1}$. Also shown are scaled (i.e., arbitrarily renormalized) radial trends for the cross-sectional area $A$ (blue dashed curve) and the ambient density $\rho_{e}$ (green dot-dashed curve). For comparison, Doppler-dimming wind-speed measurements from UVCS/SOHO (Cranmer 2020a,b) are also shown, with median values (black dotted curve) shown with \pm 1 standard deviation uncertainty bounds (gray region).

in the vicinity of relevant radii for the COR2 parcels. For this model, the Alfvén radius is located at $r_{\mathrm{A}}=10.73 R_{\odot}$, which is notably below the initial-condition parcel radius of 12.4 $R_{\odot}$. This means that a parcel that flows back down to the Sun from 12.4 $R_{\odot}$ must have an initial speed that is locally faster than the Alfvén speed. Some implications of this situation are discussed in Section 5, but for now we will assume that parcels can flow back toward the Sun at all radii $\leq 12.4 R_{\odot}$.

A numerical code was developed to integrate Equations (1) and (2) forward in time using first-order Euler steps of size $\Delta t=10^{-4} R_{\odot} /\left|u_{i, 0}\right|$. At each step, the current value of $r_{i}$ was used to interpolate the local values of $u_{e}, \rho_{e}$, and $A$ from the ZEPHYR model grids, and to compute the local values of $L$ and $\rho_{i}$ from the expressions given in Section 2.1. At least 10,000 trial models were constructed with randomized choices for four key parameters: (1) $C_{\mathrm{D}}$ was sampled from a uniform grid (in logarithm) between $10^{-6}$ and $10^{+1}$; (2) the shape exponent $\sigma$ was sampled from a uniform grid of values between -6 and +6 ; (3) the initial parcel length $L_{0}$ was sampled from a uniform grid (in logarithm) between $0.001 R_{\odot}$ and $0.5 R_{\odot}$; (4) the initial parcel overdensity ratio $\left(\rho_{i} / \rho_{e}\right)_{0}$ was sampled from a uniform grid (in logarithm) between $10^{-3}$ and $10^{+3}$.
For each numerical model, the values of $u_{i}$ at three specified radial distances were saved, compared with the observed inflow speeds, and used to compute a reduced $\chi^{2}$ goodnessof-fit parameter,

$$
\chi^{2}=\frac{1}{N} \sum_{j=1}^{N}\left[\frac{u_{i}\left(r_{j}\right)-u_{i, \mathrm{obs}}\left(r_{j}\right)}{\delta u_{i}}\right]^{2}
$$

where $N=3$. The sum is taken over the observations at $r_{j}=\{7,9,11\} R_{\odot}$, with corresponding velocities $u_{i, \mathrm{obs}}=$ $\{-22.86,-35.08,-56.62\} \mathrm{km} \mathrm{s}^{-1}$. A somewhat arbitrary uncertainty width of $\delta u_{i}=5 \mathrm{~km} \mathrm{~s}^{-1}$ was adopted as well; see Figure 7 of DeForest et al. (2014). If a given trial model never made it down to $7 R_{\odot}$ (i.e., if it was rapidly entrained into the outflowing solar wind like the high- $\omega_{0}$ models in Figure 3), it was assigned an artificially large value of $\chi^{2}$ as a flag to neglect that set of input parameters.

After randomly sampling the four-dimensional parameter space discussed above, we found no deceleration trajectories that ended up matching the observed trend very well. The "best" models (which exhibited a minimum $\chi^{2} \approx 6$ ) were very similar in appearance to the analytic solutions shown in Figure 3 for values of $\log \omega_{0}$ between about -6 and -5.5 . The model with the lowest $\chi^{2}$ had a value of $\omega_{0} \approx 1.7 \times 10^{-6} \mathrm{~s}^{-1}$, and the parcel reached a minimum radius of about $6.5 R_{\odot}$ before turning around and flowing out with the solar wind. Thus, even when taking into account the radial variation of background solar-wind parameters (which the analytic model above did not do) it seems that the assumption of a constant- $M_{i}$ parcel cannot explain the inflow pattern observed by DeForest et al. (2014).

In order to better match the observed deceleration trend, it is clear that $\omega_{0}$ should be allowed to vary even more than it does in the numerical models discussed in this section. Figure 3 indicates that $\omega_{0}$ should be rather large when the parcel is first released, then become smaller as the parcel reaches the lowest observed height. In other words, the magnitude of the drag acceleration $d u_{i} / d t$ must decrease over time.

\section{THE DRAG-BASED SNOWPLOW MODEL}

One obvious improvement that can be made to the dragbased model is to allow the parcel mass $\left(M_{i}\right)$ to vary as it flows through the corona. This section explores the idea that the parcel can accrete mass from solar-wind plasma ahead of it. Similar mass-gain effects have been reported for CMEs (e.g., Webb et al. 1996; Tappin 2006; DeForest et al. 2013; Feng et al. 2015), but it is still not certain whether this straightforward "snowplow" effect is the most likely explanation for the observations (Howard \& Vourlidas 2018). In any case, Tenerani et al. (2016) found that a parcel undergoing a gradual increase in density may be able to explain the inward deceleration seen by DeForest et al. (2014). Section 3.1 describes the differential equations for this snow- 
plow model, Section 3.2 gives analytic solutions for simplified background properties, and Section 3.3 explores numerical solutions.

\subsection{Governing Equations}

This model now consists of three coupled differential equations that must be solved simultaneously: Equations (1), (2), and a new equation that describes the rate of change of parcel mass. We generalize from earlier versions of this kind of snowplow (or bulldozer) equation, such as that of Tappin (2006), with

$$
\frac{d M_{i}}{d t}=\rho_{e} A C_{\mathrm{S}}\left|u_{i}-u_{e}\right|
$$

(see also Howard et al. 2007; Feng et al. 2015; Takahashi \& Shibata 2017). The constant $C_{\mathrm{S}}$ is introduced as an effective snowplow efficiency that describes what fraction of the plasma in front of the parcel is actually incorporated into it. Note that Tappin (2006) assumed $C_{\mathrm{S}}=1$, which describes the parcel gaining an incremental mass $d M_{i}$ as it traverses a radial length $d L=\left|u_{i}-u_{e}\right| d t$ in the rest frame of the fluid, thus sweeping out an incremental volume $d V=A d L$ containing particles with density $\rho_{e}$. Other values of $C_{\mathrm{S}}<1$ were considered by Takahashi \& Shibata (2017). The right-hand side of Equation (16) is always positive, so $M_{i}$ grows monotonically over time. Thus, Equation (2) says that the magnitude of the acceleration $d u_{i} / d t$ decreases over time, at least in comparison to the constant- $M_{i}$ case.

\subsection{Approximate Analytic Solutions}

As in Section 2.2, it is possible to first explore an analytic solution of the system in question (for the limiting case of constant values of $u_{e}, \rho_{e}, A$, and $L$ ) before solving the full equations numerically. In this case there are two coupled differential equations to solve; Equations (2) and (16). The same substitution of variables from Equation (7) allows the two equations to be written as

$$
\frac{d U}{d t}=-\frac{C_{\mathrm{D}} U^{2}}{Q L} \quad \text { and } \quad \frac{d Q}{d t}=\frac{C_{\mathrm{S}} U}{L} .
$$

As before, $U$ gets smaller as time increases, and now the parcel-mass proxy variable $Q$ grows larger as time increases. The two coupled first-order equations can be combined into a single second-order equation,

$$
U \frac{d^{2} U}{d t^{2}}=\left(\frac{C_{\mathrm{S}}}{C_{\mathrm{D}}}+2\right)\left(\frac{d U}{d t}\right)^{2}
$$

which has a closed-form solution of the form

$$
U(t)=U_{0}(1+\omega t)^{-1 / \gamma}
$$

with

$$
\gamma=\frac{C_{\mathrm{S}}}{C_{\mathrm{D}}}+1 \quad \text { and } \quad \omega=\frac{\gamma C_{\mathrm{D}} U_{0}}{Q_{0} L} .
$$

The full solution is completed by

$$
Q(t)=Q_{0}(1+\omega t)^{(\gamma-1) / \gamma} .
$$

Note that both initial conditions $U_{0}$ and $Q_{0}$ are positive, and $\gamma \geq 1$. The radial position of the parcel is given by integrating Equation (1),

$$
r_{i}(t)=r_{i, 0}+u_{e} t-\frac{Q_{0} L}{C_{\mathrm{S}}}\left[(1+\omega t)^{(\gamma-1) / \gamma}-1\right]
$$

where the same sign convention as in Equation (11) was used. The solutions from Section 2.2 are recovered exactly in the limit of $\gamma=1$, which is equivalent to $C_{\mathrm{S}}=0$ (see also Mahajan 2020).

Figure 5(a) shows a range of illustrative solutions. The choices for $r_{i, 0}, u_{i, 0}$, and $u_{e}$ were the same as in Section 2.2. For simplicity, we also chose $Q_{0}=1.5$ (i.e., initial density equipartition between the interior and exterior of the parcel), and $L=0.1 R_{\odot}$. In this case, it is possible to find combinations of parameters that match the DeForest et al. (2014) data very well. It was found that one specific value for the ratio of snowplow to drag coefficients (i.e., $\gamma \approx 26$ ) produced excellent agreement with the data. The best-fitting (central) curve in Figure 5(a) is for a model with $C_{\mathrm{S}}=0.03$ and $C_{\mathrm{D}}=0.0012$. The other curves were computed with $C_{\mathrm{S}}$ held fixed and $C_{\mathrm{D}}$ varied up and down by factors of (up to) two. Lower values of $C_{\mathrm{D}}$ imply weaker drag, so an inwardly moving parcel just keeps falling in toward the Sun. Higher values of $C_{\mathrm{D}}$ imply stronger drag, so the parcels are eventually swept into the outward-flowing solar wind.

For the best-fitting analytic model in Figure 5(a), the parcel flows in from $12.4 R_{\odot}$ to $7 R_{\odot}$ over a time of about 26.1 hours. However, this model exhibits a value of $\omega=$ $1.74 \times 10^{-4} \mathrm{~s}^{-1}$, or a characteristic deceleration timescale of only $1 / \omega \approx 1.6$ hours. This is not an inconsistency because the definition of $\omega$ involves only the initial conditions of the parcel. If a new inverse-timescale variable was defined using the time-variable quantities (i.e., $\omega^{\prime}=\gamma C_{\mathrm{D}} U(t) / Q(t) L$ ), the combined effects of mass-gain and deceleration reduce its value over time. By the time the parcel reaches $7 R_{\odot}$, this quantity has decreased to $\omega^{\prime} \approx 10^{-5} \mathrm{~s}^{-1}$, driven mainly by an increase in $Q(t)$ from 1.5 to 23 . The corresponding timescale $1 / \omega^{\prime}$ is thus 27.7 hours, comparable to the total transit time.

\subsection{Numerical Solutions}

The numerical integration code described in Section 2.3 was extended to solve Equations (1), (2), and (16). These models have radially varying trends in $\rho_{e}, u_{e}, A$, and $L$, given by the ZEPHYR polar coronal-hole model shown in 

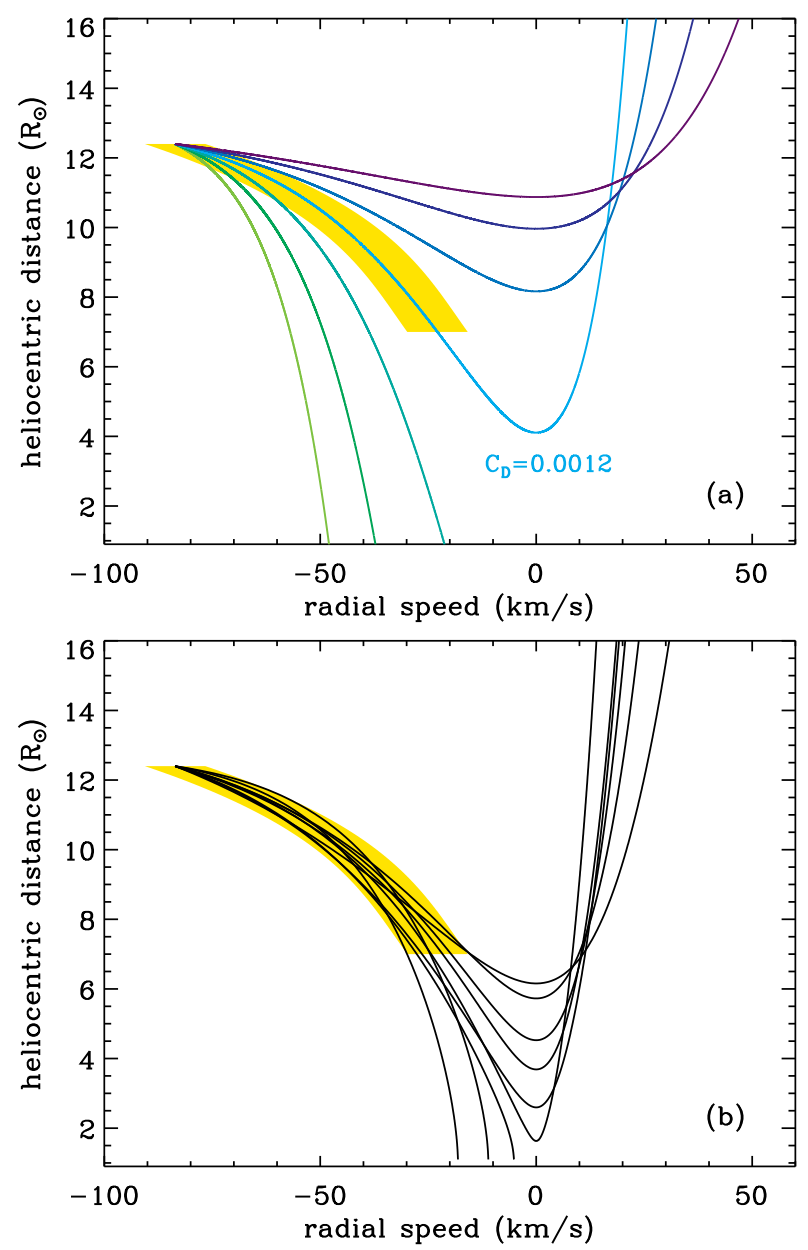

Figure 5. Analytic and numerical solutions for parcel trajectories with snowplow-like accretion. (a) Solutions to Equations (19) and (22) for $C_{\mathrm{S}}=0.03$ and a range of $C_{\mathrm{D}}$ values spaced evenly on a logarithmic grid between a minimum of $6 \times 10^{-4}$ (left-most green curve) and a maximum of $2.4 \times 10^{-3}$ (right-most violet curve). See text for other parameter values. (b) Random sample of 9 numerical solutions for radially varying background parameters, each having $\chi^{2} \leq 1$ (i.e., with parameters sampled from the histograms of Figure 6). Also shown in both panels is the observed COR2 trend from DeForest et al. (2014, yellow region).

Figure 4. Another large set of randomized trial models was constructed for the parcel motion and mass-gain, this time with different seed constants for the pseudo-random number generators than the ones used above. The parameters $\sigma, L_{0}$, and $\left(\rho_{i} / \rho_{e}\right)_{0}$ were sampled as described in Section 2.3, and in this case both coefficients $C_{\mathrm{D}}$ and $C_{\mathrm{S}}$ were sampled randomly from a uniform grid (in logarithm) between $10^{-6}$ and $10^{+1}$.

For this set of models with parcel mass-gain, there were many solutions that ended up matching the observed deceleration trend of DeForest et al. (2014). More than 10,000 total models were generated, and the code was run until a sample- size of 500 models with $\chi^{2} \leq 1$ was accumulated. The smallest value of $\chi^{2}$ in that sample was 0.059. Figure 5(b) shows nine sample trajectories of solutions with $\chi^{2} \leq 1$. Note the large variety in the possible trajectories-from parcels that turn around just below the minimum observed height of $7 R_{\odot}$, to ones that keep flowing monotonically down to the solar surface-that are all reasonably consistent with the observed velocities.

Figure 6 shows histograms of the input parameters for the set of 500 solutions with $\chi^{2} \leq 1$. The ranges of good-fitting values for $\gamma$ (which is essentially the ratio of snowplow to drag coefficients) and $\omega$ (the inverse deceleration timescale) were found to be rather narrow, with median values of $\gamma=$ 27.3 and $\omega=6.62 \times 10^{-6} \mathrm{~s}^{-1}$. The former value is almost identical to the best-fitting value of $\gamma$ found for the analytic models described in Section 3.2. For the numerical models, the drag coefficient $C_{\mathrm{D}}$ also seemed to prefer intermediate values (with a median of $C_{\mathrm{D}}=5.56 \times 10^{-4}$ ), though the distribution of values matching the observations was broader than in the cases of $\gamma$ and $\omega$. The distribution of input values for $C_{\mathrm{S}}$ is not shown, but it had a median value of 0.0135 . The combination of (more tightly constrainted) medians for $\gamma$ and $C_{\mathrm{D}}$ point to a slightly higher most-likely value of $C_{\mathrm{S}} \approx$ 0.0146 .

It is evident from Figure 6 that there do not seem to be any preferred values for $\sigma, L_{0}$, or $\rho_{i} / \rho_{e}$. Models that agree with the observed pattern of deceleration appear to be possible for nearly any of these input parameters. However, if we apply an independent estimate for a likely range of values for $L_{0}$ (say, 0.01 to $0.1 R_{\odot}$ ) it is then possible to use the medians for $C_{\mathrm{D}}$ and $\omega$ to obtain a similarly likely range of values for the overdensity parameter $Q_{0}$ between about 20 and 200.

Additional information about how much snowplowing occurs in these models can be found in Figure 7. Figure 7(a) shows the monotonic gains in parcel mass $M_{i}$ for a random subset of 50 models with $\chi^{2} \leq 1$. At the lowest COR2 height of $7 R_{\odot}$, these models exhibit a large range of final-to-initial mass ratios; i.e., between 5 and 7,000, with a median ratio of about 30. Note that for the larger set of "bad" models (i.e., with $\chi^{2}>1$; not shown) in which the parcels made it down to $7 R_{\odot}$, the distribution of mass-gain ratios was much broader: sometimes as low as 1.0001 , sometimes as high as $10^{5}$. Figure 7(b) shows how $\rho_{i} / \rho_{e}$ evolves for the same subset of models from Figure 7(a). Note that the initial range of $10^{-3}$ to $10^{+3}$ expands gradually as the parcels evolve from 12.4 to $7 R_{\odot}$. Due to the broad range of randomly chosen $\sigma$ exponents (which controls the evolution of parcel volume), there is no strong correlation between the mass-gain ratio and the local overdensity ratio.

It remains to be determined why the best-fitting values of both $C_{\mathrm{D}}$ and $C_{\mathrm{S}}$ fall so far below the order-unity expectations for these dimensionless coefficients. For the drag coefficient, 

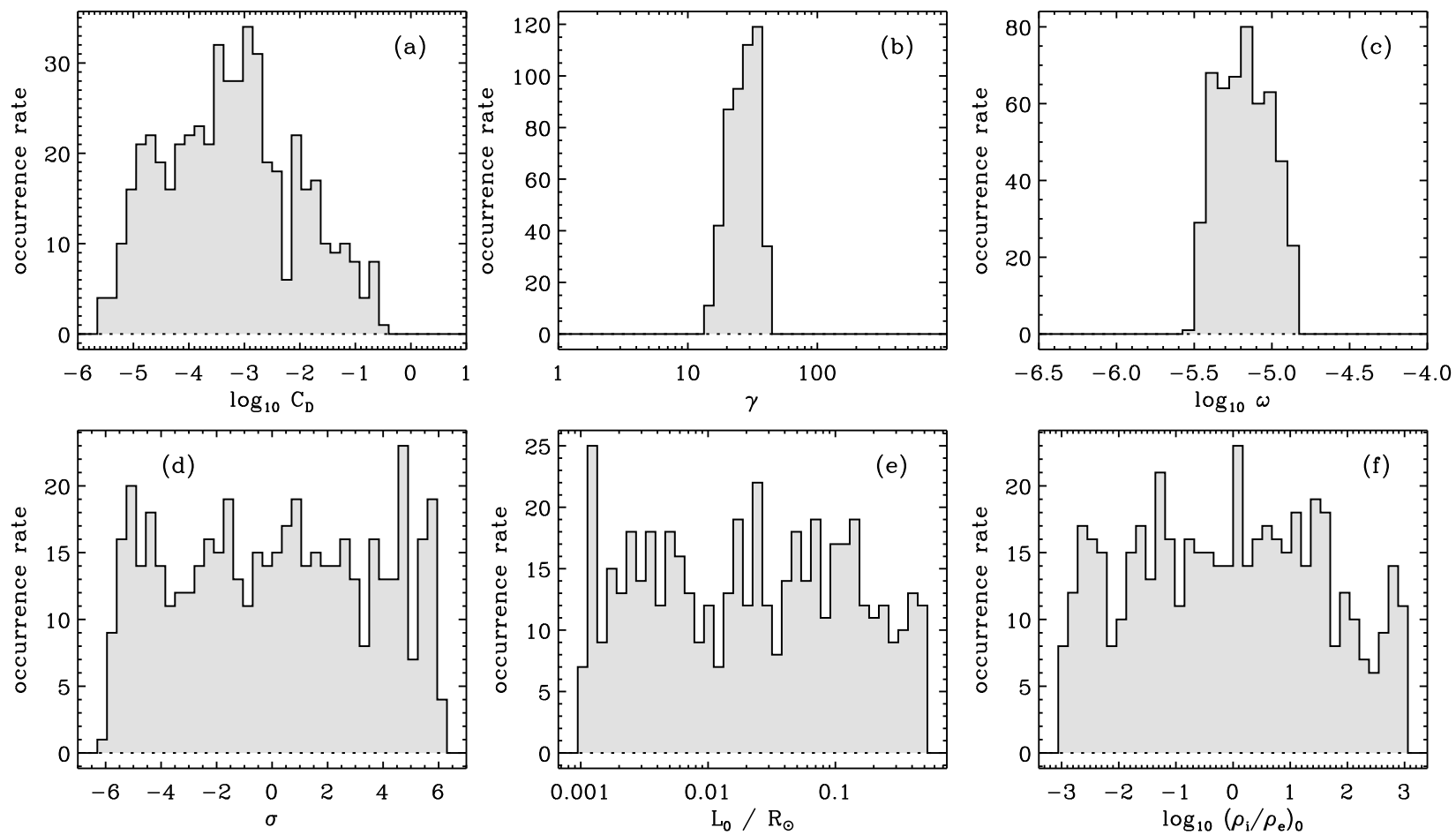

Figure 6. Histograms of probability distributions for input parameters of numerical models that agree with the DeForest et al. (2014) inflow speeds at a level of $\chi^{2} \leq 1$. Panels provide distributions for (a) $C_{\mathrm{D}}$, (b) $\gamma$, (c) $\omega$, (d) $\sigma$, (e) $L_{0}$, and (f) $\left(\rho_{i} / \rho_{e}\right)_{0}$.

it is possible that its small value can be understood as an effect of magnetic tension. Cargill et al. (1996) found that a horizontal flux rope, with an axial field pointing along the $x$-axis and rising along the $z$-axis, experiences strong turbulent drag with $C_{\mathrm{D}} \approx 1$. However, if the $z$-component of the field becomes strong enough to exceed a significant fraction of the flux rope's axial field, the vertical magnetic tension suppresses turbulent eddy-like vortices from forming behind the object, and reduces $C_{\mathrm{D}}$ to values near zero. Conceptually, this may be similar to the reduction in drag when a blunt solid object (such as a sphere) is streamlined by lengthening and tapering its surface in the downwind direction. In both cases, trailing streamlines become stretched out more along the direction of motion, and the volume of the turbulent wake behind the object is reduced drastically. For high-Reynolds-number hydrodynamic flows, it is common to see reductions in $C_{\mathrm{D}}$ from values of order 1 to values of order 0.001 (Hoerner 1965; Munson et al. 2009). Thus, a similar reduction in drag may occur for the parcels considered here. Although we have no firm constraints on their internal magnetic geometry, they do seem to be moving parallel to a strong background field as in the high- $B_{z}$ cases of Cargill et al. (1996).

\section{THE DRAG-BASED ABLATION MODEL}

If we want to explore models of plasma parcels with a time-evolving mass, it is prudent to consider mechanisms of mass loss as well as mass gain. It has been suggested that CME flux ropes (the primary inspiration for the snowplow models discussed above) may also undergo gradual erosion due to magnetic reconnection at their edges (Cargill et al. 1996; Ruffenach et al. 2015; Pal et al. 2020). In convectively unstable stellar interiors, there is also the concept of a mixing-length parcel that propagates semi-coherently until it disintegrates, thereby losing its identity and merging into the background medium. Mass-losing plasma "bullets" have also been proposed to explain thin observed filaments in the vicinity of luminous variable stars such as $\eta$ Car (Redman et al. 2002). Lastly, solid bodies such as meteors and planetesimals undergo ablative mass loss as they enter the Earth's atmosphere (Öpik 1958; Baldwin \& Sheaffer 1971; Bronshten 1983) or are accreted by protoplanets (e.g., Mordasini et al. 2016). Section 4.1 describes the differential equations for a proposed model of parcel ablation, Section 4.2 explores analytic solutions, and Section 4.3 briefly discusses numerical models.

\subsection{Governing Equations}

Because the mass-gain mechanism assumed in Section 3 was just a local snowplow, the rate of gain was straightforwardly proportional to the relative velocity $u_{i}-u_{e}$. However, for ablative mass loss, it is usually assumed that the ram pressure (i.e., kinetic energy density) of the relative flow is the primary driver of the interaction. Thus, the mass-loss 

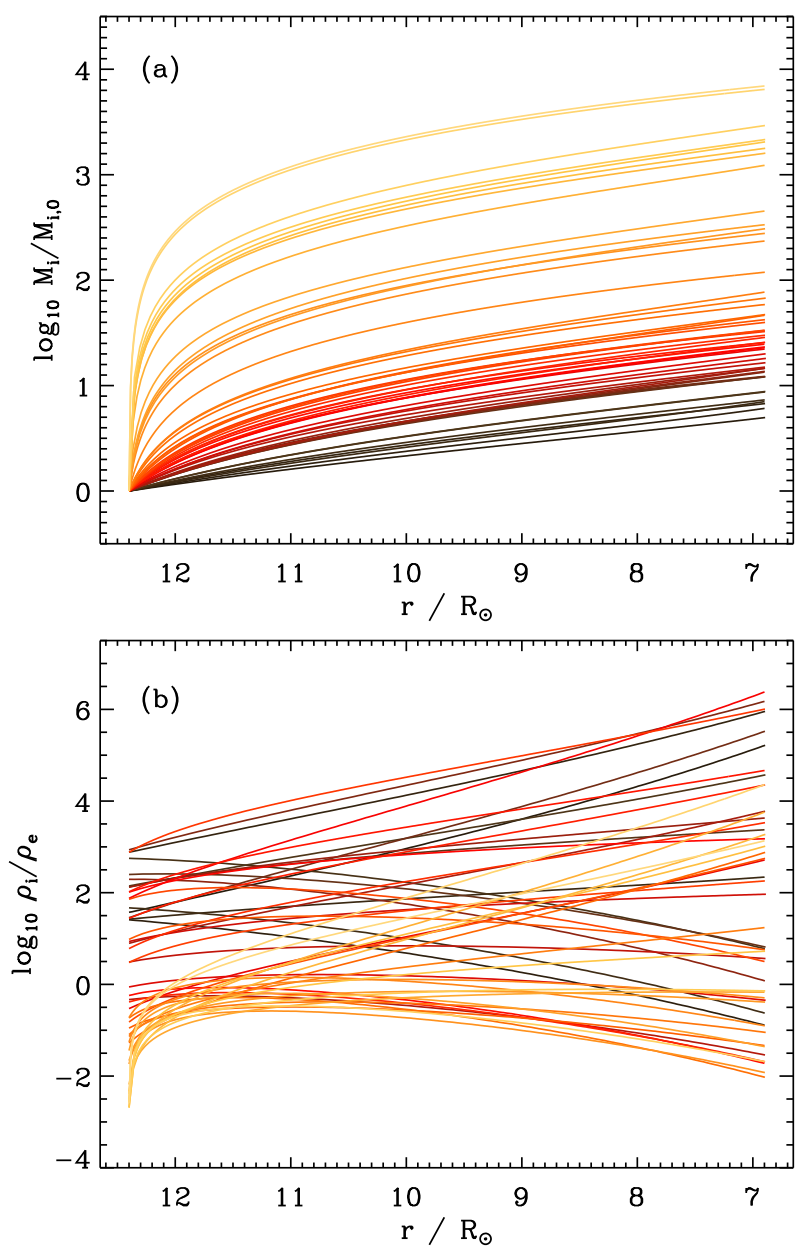

Figure 7. (a) Radial dependence of the ratio of instantaneous parcel mass $M_{i}$ to its initial mass $M_{i, 0}$ at $r=12.4 R_{\odot}$, for 50 numerical models with $\chi^{2} \leq 1$. Curve color is proportional to the final ratio at $r=7 R_{\odot}$. (b) Radial dependence of the ratio of parcel density $\rho_{i}$ to surrounding solar-wind density $\rho_{e}$ for the same set of models. Each model's curve color is taken from panel (a).

rate is proportional to $\left(u_{i}-u_{e}\right)^{3}$. We use a similar formula to that given by Bronshten (1983),

$$
\frac{d M_{i}}{d t}=-\rho_{e} A \frac{\left|u_{i}-u_{e}\right|^{3}}{V_{\mathrm{eff}}^{2}} \mathcal{H}\left(M_{i}-M_{e}\right)
$$

where $V_{\text {eff }}$ is a velocity-like quantity that describes the efficiency of the ablation. Note the presence of a Heaviside step function $\mathcal{H}\left(M_{i}-M_{e}\right)$ on the right-hand side. This constrains the ablative mass loss to occur only when $M_{i}>M_{e}$. The process is assumed to shut off completely when $M_{i}<M_{e}$; i.e., when the parcel's inertia is totally dominated by that of the surrounding solar wind.

Because there does not seem to be any well-developed theory for the ablation of plasma parcels in the solar wind, we treat the ablation effiency $V_{\text {eff }}$ as a constant free parameter.
We also speculate about a variety of approaches to estimating its value:

1. For solid meteors entering a planetary atmosphere, $V_{\text {eff }}^{2}$ is specified as a latent heat of ablation (with its velocity-squared units more typically reported as energy per unit mass). For rocky and metallic meteoric material, $V_{\text {eff }} \approx 1-3 \mathrm{~km} / \mathrm{s}$ (Baldwin \& Sheaffer 1971; Chyba et al. 1993). These particular values are not likely to be relevant for plasma parcels in the solar wind, but we find it interesting to note the parallels with this other application.

2. For astrophysical ram-pressure stripping-which efficiently removes mass from regions with gas pressure less than the large-scale flow's ram pressure-it may be appropriate to assume that $V_{\text {eff }} \approx c_{s}$, the adiabatic sound speed of the gas. For the solar corona, this hints at values around $150 \mathrm{~km} \mathrm{~s}^{-1}$.

3. Another way to estimate $V_{\text {eff }}$ is to examine hydrodynamic models of large interstellar clouds that are destroyed when they encounter shocks from nearby supernovae (Cowie \& McKee 1977; Klein et al. 1994). Klein et al. (1994) estimated that an initially spherical parcel would be mostly destroyed over a cloudcrushing timescale

$$
\tau_{c c} \approx \sqrt{\frac{\rho_{i}}{\rho_{e}}} \frac{L}{\left|u_{i}-u_{e}\right|}
$$

where we have converted the original expressions into the notation of this paper. However, another way to estimate the timescale over which a parcel undergoes substantial mass loss would be

$$
\tau_{m \ell}=\frac{M_{i}}{\left|d M_{i} / d t\right|}=\left(\frac{\rho_{i}}{\rho_{e}}\right) \frac{L V_{\mathrm{eff}}^{2}}{\left|u_{i}-u_{e}\right|^{3}}
$$

using Equation (23). Setting $\tau_{c c}=\tau_{m \ell}$, we can solve for the ablation efficiency parameter

$$
V_{\mathrm{eff}} \approx\left|u_{i}-u_{e}\right|\left(\frac{\rho_{i}}{\rho_{e}}\right)^{-1 / 4}
$$

which is never far from just $\left|u_{i}-u_{e}\right|$ itself; i.e., a few hundred $\mathrm{km} \mathrm{s}^{-1}$ for our coronal parcels.

\subsection{Approximate Analytic Solutions}

Following Sections 2.2 and 3.2, we describe a simplified system in which $u_{e}, \rho_{e}, A$, and $L$ are assumed to be constants. Using the same dependent variables as above, there are two coupled differential equations,

$$
\frac{d U}{d t}=-\frac{C_{\mathrm{D}} U^{2}}{Q L} \quad \text { and } \quad \frac{d Q}{d t}=-\frac{U^{3}}{L V_{\mathrm{eff}}^{2}} .
$$


Inspired by the mathematical technique described by Bronshten (1983), we divide the $d Q / d t$ equation by the $d U / d t$ equation. This gives a first-order separable equation for $Q$ as a function of $U$, which integrates to

$$
Q=Q_{0} \exp \left(\frac{U^{2}-U_{0}^{2}}{2 C_{\mathrm{D}} V_{\mathrm{eff}}^{2}}\right)
$$

As time increases, $U$ decreases, so $Q$ also decreases from its initial value of $Q_{0}$ to a final asymptotic value

$$
Q_{\infty}=Q_{0} \exp \left(\frac{-U_{0}^{2}}{2 C_{\mathrm{D}} V_{\mathrm{eff}}^{2}}\right)
$$

However, the above solution may not apply for all time. Under the present set of approximations, when $Q$ first becomes equal to $3 / 2$, it means that $M_{i}=M_{e}$ and the Heaviside step function in Equation (23) becomes equal to zero. Thus, once $Q$ reaches a value of $3 / 2$, it remains fixed at this value for all future times. In that case, $U(t)$ behaves like the massconserving model (Equation (9)) during this later phase of parcel evolution.

During the times when $M_{i}>M_{e}$ and ablation occurs, Equation (28) can be inserted back into Equation (27) and integrated again. There does not appear to be a closedform solution for $U(t)$, but an inverted solution for $t(U)$ can be written. If we define a new dimensionless variable $y^{2}=U^{2} /\left(2 C_{\mathrm{D}} V_{\text {eff }}^{2}\right)$, then

$$
\Omega t=\sqrt{\pi}\left[\operatorname{erfi}\left(y_{0}\right)-\operatorname{erfi}(y)\right]+\frac{e^{y^{2}}}{y}-\frac{e^{y_{0}^{2}}}{y_{0}}
$$

where

$$
\Omega=\frac{2^{1 / 2} C_{\mathrm{D}}^{3 / 2} V_{\mathrm{eff}}}{Q_{\infty} L}
$$

and the imaginary error function $\operatorname{erfi}(z)=-i \operatorname{erf}(i z)$ is realvalued for a real argument $z$. In the weak-ablation limit of $V_{\text {eff }} \rightarrow \infty$, Equation (30) reduces to Equation (9) as it should.

\subsection{Numerical Solutions}

As in Sections 2.3 and 3.3, a large set of numerical models was constructed to determine if any combinations of parameters could reproduce the observed deceleration trend of DeForest et al. (2014). In this case, similar ranges of random values for $C_{\mathrm{D}}, \sigma$, and $L_{0}$ were explored. The initial parcel density ratio $\left(\rho_{i} / \rho_{e}\right)_{0}$ was sampled from a smaller range of 1.001 to $10^{+3}$; i.e., excluding values less than unity that would not exhibit any ablation. The efficiency parameter $V_{\text {eff }}$ was sampled randomly from a uniform grid (in logarithm) over twelve orders of magnitude: from $10^{-6} c_{s}$ to $10^{+6} c_{s}$, with a fiducial value of the coronal sound speed $c_{s}=150$ $\mathrm{km} \mathrm{s}^{-1}$.
Unfortunately, there were no combinations of input parameters that agreed with the observed deceleration trend. The "best" models were ones with extremely weak ablation (i.e., the largest values of $V_{\text {eff }}$ ), and these were virtually identical to the mass-conserving models discussed in Sections 2.2-2.3. Minimum $\chi^{2}$ values were similarly around 6 , for minimum radii of about $6.5 R_{\odot}$ and typical values of $\omega_{0} \approx 1.7 \times 10^{-6}$ $\mathrm{s}^{-1}$. Many of the ablated solutions ended up with overdensity ratios $\rho_{i} / \rho_{e} \lesssim 1$ for $r_{i}$ between 7 and $12 R_{\odot}$. Parcels of this kind may not exhibit enough scattered-light contrast to ever be visible with instruments like COR2, so it is probably not surprising that no parcels with these kinematic properties were observed.

\section{OBSERVATIONAL CONSTRAINTS ON INITIAL CONDITIONS}

The snowplow model of Section 3 appears to be able to reproduce the observed deceleration trend of inflowing parcels in the extended corona. However, we have not addressed the origin of the assumed initial condition of $u_{i, 0}=-83.6$ $\mathrm{km} \mathrm{s}^{-1}$ at $r_{i, 0}=12.4 R_{\odot}$. For the 11 days of COR2 observations analyzed by DeForest et al. (2014), there are several possibilities: (1) that $r_{i, 0}$ was consistently below the Alfvén radius, in which case it is possible for the parcels to be moving at either Alfvénic or sub-Alfvénic speeds in the frame of the solar wind, (2) that $r_{i, 0}$ remained above the Alfvén radius, in which case inward-moving parcels must have been "shot out of a cannon" at a locally supra-Alfvénic speed, (3) that the Alfvén radius drifted in radius over those 11 days so that both possibilities (1) and (2) applied over some of that time, or (4) the solar wind exhibited multiple Alfvén radii during this time. Nontrivial situations like (3) and (4) are discussed further in Section 6.

In the remainder of this section, we use in situ data to estimate an expected range of downward flow speeds for coronal perturbations at $12.4 R_{\odot}$. First, it is necessary to estimate the value of $r_{\mathrm{A}}$ itself for a given set of heliospheric conditions. There have been several proposed empirical methods for using data at $1 \mathrm{AU}$ to estimate the radial distance of the Alfvén surface (see, e.g., Katsikas et al. 2010; Zhao \& Hoeksema 2010; Goelzer et al. 2014; Tasnim \& Cairns 2016; Kasper \& Klein 2019; Liu et al. 2021). In steady-state, one can combine the equations of mass and magnetic flux conservation to see that the quantity $\rho u^{2} / V_{\mathrm{A} r}^{2}$ should remain constant along a magnetic field line. We refer to Equation (14) to note that the Alfvén speed $V_{\mathrm{A} r}$ is defined here using only the radial component of the magnetic field $B_{r}$, and we now use $u$ to refer to the large-scale wind speed external to the parcel, rather than $u_{e}$ as above. Thus, because $u^{2} / V_{\mathrm{A} r}^{2}=1$ at the Alfvén radius, we see that

$$
\frac{\rho_{\mathrm{A}}}{\rho_{\mathrm{E}}}=\left(\frac{u}{V_{\mathrm{A} r}}\right)_{\mathrm{E}}^{2}
$$


where subscript A refers to the Alfvén radius and subscript $\mathrm{E}$ refers to the Earth at $1 \mathrm{AU}$. Obtaining a measurement of the right-hand side of Equation (32) is straightforward, and it constrains the ratio of the density at the Alfvén radius to the density at $1 \mathrm{AU}$.

The density ratio described above can be converted into a heliocentric radial distance by examining models of solar-wind acceleration. We took a large database of one-dimensional ZEPHYR models-i.e., 30 models from Cranmer et al. (2007) and 289 models from Cranmer et al. (2013) — and produced distributions of the radii corresponding to specific values of $\rho_{\mathrm{A}} / \rho_{\mathrm{E}}$. The models from Cranmer et al. (2007) were created with idealized magnetic fields corresponding to coronal holes, helmet streamers, and the open-field parts of active regions. The models from Cranmer et al. (2013) were created by performing a potential-field extrapolation from a near-equatorial patch of quiet Sun observed by the the Synoptic Optical Long-term Investigations of the Sun (SOLIS) facility in 2003.

Figure 8 (a) shows the radial dependence of the median values of derived distributions of $\rho_{\mathrm{A}} / \rho_{\mathrm{E}}$, as well as the minimum and maximum radii corresponding to each value of the density ratio. For example, if Equation (32) indicates that a field-line has a value of $\rho_{\mathrm{A}} / \rho_{\mathrm{E}}=10^{3}$, one can infer from the ZEPHYR models that this occurs at a median radius $r_{\mathrm{A}}=8.9 R_{\odot}$, with a relatively small spread around that value of $8.4-9.3 R_{\odot}$.

Tasnim \& Cairns (2016) proposed a more straightforward approximation for finding a direct solution of Equation (32). If the Alfvén radius is large enough that negligible solar-wind acceleration occurs above it, then one can assume $u=$ constant. Far enough from the Sun, this implies $\rho \propto r^{-2}$, so the left-hand side of Equation (32) can be replaced simply by $\left(r_{\mathrm{E}} / r_{\mathrm{A}}\right)^{2}$, where $r_{\mathrm{E}}=1 \mathrm{AU}$, and the expression can be solved analytically for $r_{\mathrm{A}}$. Figure 8(a) shows how this constant wind-speed model provides a slightly smaller value of $r_{\mathrm{A}}$, for any given measured density ratio $\rho_{\mathrm{A}} / \rho_{\mathrm{E}}$, than do the ZEPHYR models that include solar-wind acceleration.

Ideally, we would prefer to carry out these measurements for the specific solar-wind streams connected to the polar coronal holes observed by DeForest et al. (2014) in August 2007. However, it is not clear whether these regions were magnetically connected to any spacecraft in the ecliptic plane or elsewhere in the solar system (e.g., Ulysses). Thus, for the present analysis, we chose to examine a full solar cycle's worth of OMNI data at 1 AU (calendar years 2008-2018) in order to sample as many high-speed wind regions as possible that may have been connected to large coronal holes.

One-minute-cadence OMNI data were used as a starting point (see, e.g., King \& Papitashvili 2005), then we extracted mean values of $u$ and $V_{\mathrm{A} r}$ in successive two-hour bins. With perfect data, that would provide 48,216 samples over the se-
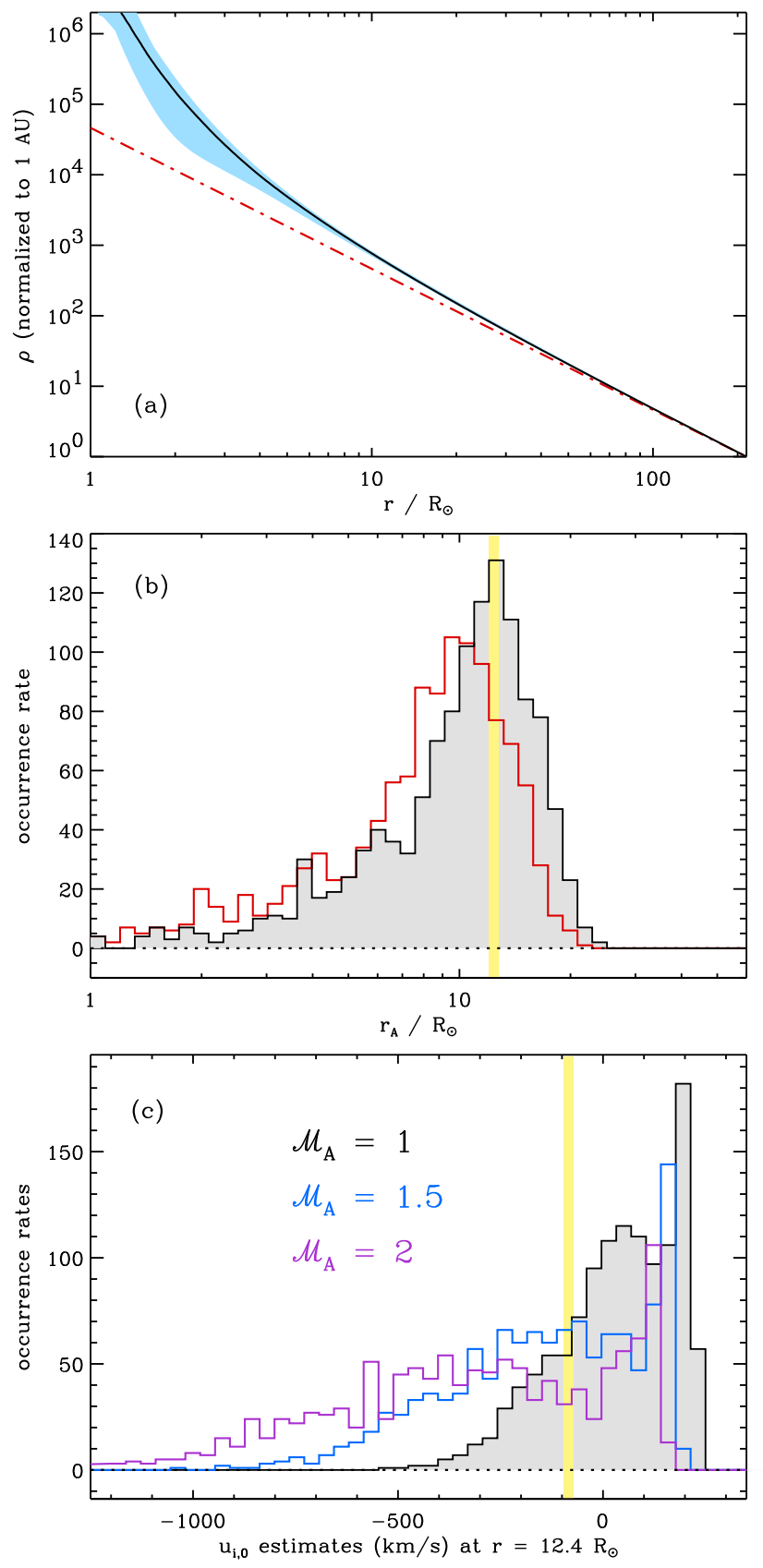

Figure 8. (a) Ratio of density $\rho$ at various radii to its value at $1 \mathrm{AU}$, computed from a constant wind-speed model (red dot-dashed curve), from the median of a set of ZEPHYR models (black solid curve), and from the full range of ZEPHYR models (light-blue region). (b) Histogram of values for $r_{\mathrm{A}}$ computed from OMNI fastwind data, in combination with the median ZEPHYR model (black curve) and the constant wind-speed model (red curve). Initial parcel radius $r_{i, 0}$ is shown as a yellow bar. (c) Histogram of inwardcharacteristic speeds computed from Equation (35) using Alfvén Mach numbers $\mathcal{M}_{\mathrm{A}}=1$ (black curve), 1.5 (blue curve), and 2 (purple curve), compared with the observed initial parcel speed $u_{i, 0}$ (yellow bar). 
lected 11-year period. However, there were frequent data gaps (which were identified easily) and CMEs (which were identified using the criteria of Xu \& Borovsky 2015). In order to isolate the data that accurately sample the ambient solar wind, we rejected any bin containing more than 40 oneminute data points (out of 120) with bad data or CMEs. This reduced the total sample to 23,172 bins. We then kept only high-speed wind data (appropriate for large coronal holes) with radial speeds between 600 and $900 \mathrm{~km} \mathrm{~s}^{-1}$, which reduced the sample further to only 1,208 bins.

Figure 8(b) shows the result of converting the measured distribution of density ratios to values of $r_{\mathrm{A}}$. When using the median ZEPHYR density trend, the median value of $r_{\mathrm{A}}$ was $10.9 R_{\odot}$, which is close to the value of $10.73 R_{\odot}$ corresponding to the baseline coronal-hole model shown in Figure 4. For the distribution of ZEPHYR-derived results for $r_{\mathrm{A}}, 64 \%$ of the values fall below the initial parcel radius $r_{i, 0}=12.4 R_{\odot}$. When using the Tasnim \& Cairns (2016) density trend, the median value of $r_{\mathrm{A}}$ was $8.7 R_{\odot}$, and $81 \%$ of the data correspond to $r_{\mathrm{A}}<12.4 R_{\odot}$.

Because $r_{\mathrm{A}}$ is sometimes larger than $12.4 R_{\odot}$ and sometimes it is smaller, it is necessary to extrapolate from $r_{\mathrm{A}}$ both up and down in order to estimate the initial speeds of parcels. Our goal is to first compute the linear characteristic speeds of radial Alfvénic perturbations $\left(C_{ \pm}=u \pm V_{\mathrm{A} r}\right)$ at values of $r_{i, 0}$ that may be above or below $r_{\mathrm{A}}$. The OMNI measurement of radial wind speed $1 \mathrm{AU}$ can be used in combination with mass-flux conservation to estimate the value of $u=V_{\mathrm{A} r}$ at the Alfvén radius,

$$
u_{\mathrm{A}} \approx u_{\mathrm{E}}\left(\frac{\rho_{\mathrm{E}}}{\rho_{\mathrm{A}}}\right)\left(\frac{r_{\mathrm{E}}}{r_{\mathrm{A}}}\right)^{2}
$$

where we assume that there is no superradial expansion between $r_{\mathrm{A}}$ and $1 \mathrm{AU}$ (i.e., $A \propto r^{2}$ ). For the fast-wind OMNI data, the median value obtained with the ZEPHYR density ratios was $u_{\mathrm{A}}=403 \mathrm{~km} \mathrm{~s}^{-1}$, with a large standard deviation of about $100 \mathrm{~km} \mathrm{~s}^{-1}$ around that value. For comparison, the ZEPHYR model shown in Figure 4 had $u_{\mathrm{A}}=509 \mathrm{~km} \mathrm{~s}^{-1}$.

In order to extrapolate up and down in the neighborhood of the Alfvén radius, we can approximate $A \propto r^{p}$ and $\rho \propto$ $r^{-q}$. In Section 2.1, values of $p \approx 2.15$ and $q \approx 2.45$ were provided for the baseline coronal-hole model between 7 and $12 R_{\odot}$. Thus, mass-flux conservation and the definition of the Alfvén speed give

$$
u \propto r^{q-p} \quad \text { and } \quad V_{\mathrm{A} r} \propto r^{(q-2 p) / 2} .
$$

These relations allow us to estimate the characteristic speeds of parcels that exist at radii other than $r_{\mathrm{A}}$. Here we focus solely on the downward mode, and we allow for the possibility of nonlinear propagation. Thus, we can write $C_{-}=$ $u-\mathcal{M}_{\mathrm{A}} V_{\mathrm{A} r}$, where an Alfvénic Mach number $\mathcal{M}_{\mathrm{A}}=1$ indicates linear wave motion and $\mathcal{M}_{\mathrm{A}}>1$ indicates supraAlfvénic (possibly shock-driven) motion, and

$$
C_{-}(r)=u_{\mathrm{A}}\left[\left(\frac{r}{r_{\mathrm{A}}}\right)^{q-p}-\mathcal{M}_{\mathrm{A}}\left(\frac{r}{r_{\mathrm{A}}}\right)^{(q-2 p) / 2}\right] \text {. }
$$

Figure 8(c) shows the resulting distributions of $C_{-}$speeds computed at a radial distance of $r_{i, 0}=12.4 R_{\odot}$, using the ZEPHYR-derived results for $r_{\mathrm{A}}$ and three choices for $\mathcal{M}_{\mathrm{A}}$.

For the linear case $\left(\mathcal{M}_{\mathrm{A}}=1\right)$, the median value of the downward characteristic speed is positive (i.e., $+53 \mathrm{~km} \mathrm{~s}^{-1}$ ) since this situation corresponds to the initial parcel radius $r_{i, 0}=12.4 R_{\odot}$ sitting above the median Alfvén radius $r_{\mathrm{A}} \approx 10.9 R_{\odot}$. For the baseline distribution shown in Figure 8(c), downward characteristic speeds of $C_{-} \leq-83.6$ $\mathrm{km} \mathrm{s}^{-1}$ occur only in $21 \%$ of the samples. However, Equation (35) shows that it is possible to shift the distribution of $C_{-}$to smaller (more negative) values by increasing $\mathcal{M}_{\mathrm{A}}$. In fact, the measured downward speed of $-83.6 \mathrm{~km} \mathrm{~s}^{-1}$ can be obtained as the median of the distribution when $\mathcal{M}_{\mathrm{A}}$ is increased to a value of roughly 1.4. The trend for the values of $C_{-}$to decrease as $\mathcal{M}_{\mathrm{A}}$ is increased is captured by a linear fit of these results,

$$
\left\langle C_{-}\left(r_{i, 0}\right)\right\rangle \approx\left(413-355 \mathcal{M}_{\mathrm{A}}\right) \mathrm{km} \mathrm{s}^{-1}
$$

where the angle-brackets denote median values of the distributions constructed for each choice of $\mathcal{M}_{\mathrm{A}}$. Note that only a slightly nonlinear Mach number $\left(\mathcal{M}_{\mathrm{A}} \approx 1.17\right)$ is needed to produce a median downward characteristic speed of zero at $12.4 R_{\odot}$.

Is it possible that the initial downward speeds of the parcels measured by DeForest et al. (2014) are actually supra-Alfvénic in the frame of the accelerating solar wind? There are models of collisionless magnetic reconnection in which the exhausts are affected by kinetic process and accelerated to speeds higher than the local Alfvén speed (e.g., Shay et al. 2011; Lapenta et al. 2013; Lu et al. 2014). However, in cases where the Hall effect is responsible for these rapid flows, the relevant spatial scales may be far smaller than those of the observed parcels. Models of jets formed by reconnection in the low corona often exhibit either slow-mode or fast-mode MHD shocks (Yokoyama \& Shibata 1996; Yang et al. 2013; Roberts et al. 2018), but in some cases the absolute speeds may be lower than $V_{\mathrm{A} r}$ due to guide-field reconnection ignoring much of the radial field.

The recent discovery of sharp magnetic "switchbacks" in the inner heliosphere by Parker Solar Probe may point to the ubiquitous presence of highly nonlinear MHD structures in the solar-wind acceleration region (Bale et al. 2019; Kasper et al. 2019; Dudok de Wit et al. 2020; Horbury et al. 2020). In fact, the list of proposed explanations for switchbacks sounds very similar to the list of origin scenarios for 
inward-propagating coronal parcels. Phenomena such as magnetic reconnection, shear-driven instabilities, and largeamplitude turbulent eddies have all been suggested (e.g., Squire et al. 2020; Tenerani et al. 2020; Ruffolo et al. 2020; Zank et al. 2020; Shoda et al. 2021). It may be the case that the same events produce both outward and inward disturbances.

\section{DISCUSSION AND CONCLUSIONS}

Inspired by observations of decelerating inflows at heliocentric distances between 7 and $12 R_{\odot}$, we constructed dynamical models of discrete plasma parcels in the corona. We found that parcels with constant or decreasing mass are not able to reproduce the pattern of deceleration observed by DeForest et al. (2014). However, parcels with increasing mass-i.e., undergoing snowplow-like interactions with the surrounding solar-wind plasma-can reproduce the observed deceleration pattern (see also Tenerani et al. 2016). We also found that the most likely initial conditions for these parcels at $\sim 12 R_{\odot}$ involve mildly nonlinear (i.e., supra-Alfvénic) speeds like those associated with shocks or jets.

Although the models developed in this paper help point us to the most important physical processes at work, it will be useful to supplement them with more realistic simulations of parcels associated with, e.g., magnetic reconnection, KelvinHelmholtz instabilities, or MHD turbulence. For example, Lynch (2020) simulated inflows at 2-6 $R_{\odot}$ from intermittent magnetic reconnection at the tips of helmet streamers. Selfconsistent simulations extended to larger distances may tell us why only specific values of the drag coefficient $C_{\mathrm{D}}$ and snowplow efficiency $C_{\mathrm{S}}$ seemed applicable to the observed flow patterns. Also, some of our assumptions about geometric inertia effects (i.e., $C_{\mathrm{A}}$ ) and even the quadratic nature of the drag may need to be revised in the light of new simulations (see, e.g., Maloney \& Gallagher 2010; Verma et al. 2020).

Despite this paper's focus on hydrodynamic and (mostly ideal) MHD processes, it may be necessary to include additional physics in models of coronal inflows. The presence of strong electron heat conduction leads to the existence of collisionless thermal fronts that propagate at speeds of order $c_{s}$ and $V_{\mathrm{A}}$ but depend on the local plasma properties in different ways (see, e.g., Brown et al. 1979; Rust et al. 1985; Karlický 2015). The physics of these conduction fronts may govern the observed kinematics of polar jets seen above the limb with Hinode (Shimojo et al. 2007) and in the coronal-hole magnetic network by IRIS (Tian et al. 2014). These jets may also be related to the ubiquitous polar plumes, which are often seen to survive out to the Alfvén surface (DeForest et al. 1997; Raouafi et al. 2008, 2016).

It has been proposed that the Alfvén radius is more accurately described as being a frothy "Alfvén zone," and that at any one time there may be multiple points along a radial line at which the wind speed equals the Alfvén speed. DeForest et al. (2018) discussed how plasma parcels of different sizes may flow at different speeds in such an environment because the characteristic speeds can vary from one end of the parcel to the other. In fact, there may be places where short-wavelength parcels propagate inward and longwavelength parcels propagate outward. Also, with a complex enough three-dimensional structure, individual parcels may undergo random-walk-like deflections-alternately in and out - over their lifetimes. Thus, the steadily decelerating inflow pattern detected with STEREO/COR2 may be the result of intermittently sampling a distribution rather than tracing a laminar trajectory. In 2023, the Polarimeter to Unify the Corona and Heliosphere (PUNCH) will begin mapping low-contrast motions between 6 and $180 R_{\odot}$ (DeForest et al. 2020). This, along with other future coronagraphs and heliospheric imagers, will provide much better constraints about the dynamics and statistics of inhomogeneous flows in the solar wind.

\section{ACKNOWLEDGMENTS}

The authors gratefully acknowledge Anna Tenerani, Yuhong Fan, and the PUNCH science team for many valuable discussions. The authors are also grateful to the anonymous referee for many constructive suggestions that have improved this paper. SRC's contribution to this work was supported by the National Aeronautics and Space Administration (NASA) under grants NNX15AW33G and NNX16AG87G, and by the National Science Foundation (NSF) under grant 1613207. The National Center for Atmospheric Reseach is a major facility sponsored by the NSF under Cooperative Agreement No. 1852977. This research made extensive use of NASA's Astrophysics Data System (ADS). The authors acknowledge use of OMNI data from NASA's Space Physics Data Facility OMNIWeb service.

\section{REFERENCES}

Abbo, L., Ofman, L., Antiochos, S. K., et al. 2016, SSRv, 201, 55

Antolin, P., Vissers, G., Pereira, T. M. D., Rouppe van der Voort,

L., \& Scullion, E. 2015, ApJ, 806, 81

Baldwin, B., \& Sheaffer, Y. 1971, J. Geophys. Res., 76, 4653
Bale, S. D., Badman, S. T., Bonnell, J.W., et al. 2019, Nature, 576, 237

Belcher, J. W. 1971, ApJ, 168, 509

Belcher, J. W., \& MacGregor, K. B. 1976, ApJ, 210, 498 
Borgazzi, A., Lara, A., Echer, E., \& Alves, M. V. 2009, A\&A, 498, 885

Bretherton, F. P., \& Garrett, C. J. R. 1968, Proc. Roy. Soc. A, 302, 529

Bronshten, V. A. 1983, Physics of Meteoric Phenomena (Dordrecht: Reidel)

Brown, J. C., Melrose, D. B., \& Spicer, D. S. 1979, ApJ, 228, 592

Cargill, P. J. 2004, SoPh, 221, 135

Cargill, P. J., Chen, J., Spicer, D. S., \& Zalesak, S. T. 1996, J. Geophys. Res., 101, 4855

Chen, J. 1989, ApJ, 338, 453

Chen, J. 1996, J. Geophys. Res., 101, 27499

Chhiber, R., Usmanov, A. V., Matthaeus, W. H., \& Goldstein, M. L. 2019, ApJS, 241, 11

Chyba, C. F., Thomas, P. J., \& Zahnle, K. J. 1993, Nature, 361, 40 Cohen, O. 2015, SoPh, 290, 2245

Corless, R. M., Gonnet, G. H., Hare, D. E. G., Jeffrey, D. J., \& Knuth, D. E. 1996, Adv. Comput. Math., 5, 329

Cowie, L. L., \& McKee, C. F. 1977, ApJ, 211, 135

Cranmer, S. R. 2020a, ApJ, 900, 105

Cranmer, S. R. 2020b, Heating Rates for Protons and Electrons in

Polar Coronal Holes: Empirical Constraints from UVCS/SOHO (Associated Data), v1.0, Zenodo, doi:10.5281/zenodo.3908519

Cranmer, S. R., \& van Ballegooijen, A. A. 2005, ApJS, 156, 265

Cranmer, S. R., van Ballegooijen, A. A., \& Edgar, R. J. 2007, ApJS, 171, 520

Cranmer, S. R., van Ballegooijen, A. A., \& Woolsey, L. N. 2013, ApJ, 767, 125

DeForest, C. E., Hoeksema, J. T., Gurman, J. B., et al. 1997, SoPh, 175,393

DeForest, C. E., Howard, R. A., Velli, M., et al. 2018, ApJ, 862, 18

DeForest, C. E., Howard, T. A., \& McComas, D. J. 2013, ApJ, 769, 43

DeForest, C. E., Howard, T. A., \& McComas, D. J. 2014, ApJ, 787, 124

DeForest, C. E., Killough, R., Gibson, S. E., et al. 2020, AGU Fall Meeting, SH028-0002

DeForest, C. E., Matthaeus, W. H., Viall, N. M., \& Cranmer, S. R. 2016, ApJ, 828, 66

Dolei, S., Bemporad, A., \& Spadaro, D. 2014, A\&A, 562, A74

Dudok de Wit, T., Krasnoselskikh, V. V., Bale, S. D., et al. 2020, ApJS, 246, 39

Dumbović, M., Čalogović, J., Vršnak, B., et al. 2018, ApJ, 854, 180

Feng, L., Wang, Y., Shen, F., et al. 2015, ApJ, 812, 70

Fitzpatrick, R. 2017, Theoretical Fluid Mechanics (Bristol, UK: IOP Publishing)

Goelzer, M. L., Schwadron, N. A., \& Smith, C. W. 2014, J. Geophys. Res., 119, 115

Gosling, J. T. 2007, ApJL, 671, L73
Gronke, M., \& Oh, S. P. 2018, MNRAS, 480, L111

Haerendel, G., \& Berger, T. 2011, ApJ, 731, 82

Hoerner, S. F. 1965, Fluid-Dynamic Drag: Practical Information on Aerodynamic Drag and Hydrodynamic Resistance (Bakersfield, CA: Hoerner Fluid Dynamics)

Horbury, T. S., Woolley, T., Laker, R., et al. 2020, ApJS, 246, 45

Howard, R. A., Moses, J. D., Vourlidas, A., et al. 2008, SSRv, 136, 67

Howard, R. A., \& Vourlidas, A. 2018, SoPh, 293, 55

Howard, T. A., Fry, C. D., Johnston, J. C., \& Webb, D. F. 2007, ApJ, 667, 610

Karlický, M. 2015, ApJ, 814, 153

Kasper, J. C., Bale, S. D., Belcher, J.W., et al. 2019, Nature, 576, 228

Kasper, J. C., \& Klein, K. G. 2019, ApJL, 877, L35

Katsikas, V., Exarhos, G., \& Moussas, X. 2010, Adv. Space Res., 46, 382

Kay, C., Mays, M. L., \& Verbeke, C. 2020, Space Weather, 18, e02382

King, J. H., \& Papitashvili, N. E. 2005, J. Geophys. Res., 110, A02104

Klein, R. I., McKee, C. F., \& Colella, P. 1994, ApJ, 420, 213

Kohl, J. L., Noci, G., Cranmer, S. R., \& Raymond, J. C. 2006, A\&A Rv, 13, 31

Lamb, H. 1932, Hydrodynamics, 6th ed. (New York: Dover)

Lapenta, G., Goldman, M., Newman, D., \& Markidis, S. 2013, Phys. Plasmas, 20, 102113

Li, Z., Hopkins, P. F., Squire, J., \& Hummels, C. 2020, MNRAS, 492, 1841

Lin, J., Cranmer, S. R., \& Farrugia, C. J. 2008, J. Geophys. Res., 113, A11107

Liu, Y. D., Chen, C., Stevens, M. L., \& Liu, M. 2021, ApJL, 908, L41

Lu, S., Lu, Q., Huang, C., et al. 2014, New J. Phys., 16, 083021

Lynch, B. J. 2020, ApJ, 905, 139

Mahajan, S. 2020, Am. J. Phys., 88, 261

Maloney, S. A., \& Gallagher, P. T. 2010, ApJL, 724, L127

McKenzie, D. E. 2000, SoPh, 195, 381

Mordasini, C., van Boekel, R., Mollière, P., et al. 2016, ApJ, 832, 41

Morel, C. 2015, Mathematical Modeling of Disperse Two-Phase Flows (New York: Springer)

Mullan, D. J. 1990, A\&A, 232, 520

Müller, D. A. N., De Groof, A., Hansteen, V. H., \& Peter, H. 2005, A\&A, 436, 1067

Munson, B. R., Young, D. F., Okiishi, T. H., \& Huebsch, W. W. 2009, Fundamentals of Fluid Mechanics, 6th ed. (Hoboken, NJ: Wiley)

Ofman, L., \& Thompson, B. J. 2011, ApJL, 734, L11 
Öpik, E. J. 1958, Physics of Meteor Flight in the Atmosphere (New York: Interscience)

Pal, S., Dash, S., \& Nandy, D. 2020, Geophys. Res. Lett., 47, e2019GL086372

Parker, E. N. 1957, ApJS, 3, 51

Pinto, R. F., Brun, A. S., Jouve, L., \& Grappin, R. 2011, ApJ, 737, 72

Pneuman, G. W., \& Cargill, P. J. 1985, ApJ, 288, 653

Raouafi, N.-E., Petrie, G. J. D., Norton, A. A., Henney, C. J., \& Solanki, S. K. 2008, ApJL, 682, L137

Raouafi, N.-E., Patsourakos, S., Pariat, E., et al. 2016, SSRv, 201, 1

Rappazzo, A. F., Matthaeus, W. H., Ruffolo, D., Servidio, S., \& Velli, M. 2012, ApJL, 758, L14

Redman, M. P., Meaburn, J., \& Holloway, A. J. 2002, MNRAS, 332,754

Réville, V., Velli, M., Rouillard, A. P., et al. 2020, ApJL, 895, L20

Roberts, D. A., Goldstein, M. L., Matthaeus, W. H., \& Ghosh, S. 1992, J. Geophys. Res., 97, 17115

Roberts, M. A., Uritsky, V. M., DeVore, C. R., \& Karpen, J. T. 2018, ApJ, 866, 14

Ruffenach, A., Lavraud, B., Farrugia, C. J., et al. 2015, J. Geophys. Res., 120, 43

Ruffolo, D., Matthaeus, W. H., Chhibber, R., et al. 2020, ApJ, 902, 94

Rust, D. M., Simnett, G. M., \& Smith, D. F. 1985, ApJ, 288, 401

Ryutova, M. P., \& Priest, E. R. 1993, ApJ, 419, 349

Sanchez-Diaz, E., Rouillard, A. P., Davies, J. A., et al. 2017, ApJL, 835, L7

Sanchez-Diaz, E., Rouillard, A. P., Lavraud, B., et al. 2019, ApJ, 882,51

Savage, S. L., McKenzie, D. E., \& Reeves, K. K. 2012, ApJL, 747, L40

Schlüter, A. 1957, in IAU Symp. 4, Radio Astronomy, ed. H. Van de Hulst (Cambridge: Cambridge Univ. Press), 356

Schwenn, R. 2006, SSRv, 124, 51

Shay, M. A., Drake, J. F., Eastwood, J. P., \& Phan, T. D. 2011, PhRvL, 107, 065001

Sheeley, N. R., \& Wang, Y.-M. 2014, ApJ, 797, 10

Sheeley, N. R., Wang, Y.-M., Hawley, S. H., et al. 1997, ApJ, 484, 472
Shimojo, M., Narukage, N., Kano, R., et al. 2007, PASJ, 59, S745

Shoda, M., Chandran, B. D. G., \& Cranmer, S. R. 2021, ApJ, submitted, arXiv:2101.09529

Simnett, G. M., 2004, A\&A, 416, 759

Spruit, H. C. 1981, A\&A, 98, 155

Squire, J., Chandran, B. D. G., \& Meyrand, R. 2020, ApJL, 891, L2

Subramanian, P., Lara, A., \& Borgazzi, A. 2012,

Geophys. Res. Lett., 39, L19107

Takahashi, T., \& Shibata, K. 2017, ApJL, 837, L17

Tappin, S. J. 2006, SoPh, 233, 233

Tasnim, S., \& Cairns, I. H. 2016, J. Geophys. Res., 121, 4966

Tenerani, A., Velli, M., \& DeForest, C. E. 2016, ApJL, 825, L3

Tenerani, A., Velli, M., Matteini, L., et al. 2020, ApJS, 246, 32

Tian, H., DeLuca, E., Cranmer, S. R., et al. 2014, Science, 346, 1255711

Tokumaru, M., Kojima, M., \& Fujiki, K. 2010, J. Geophys. Res., 115, A04102

Verma, M. K., Alam, S., \& Chatterjee, S. 2020, Phys. Plasmas, 27, 052301

Vršnak, B. 2001, SoPh, 202, 173

Vršnak, B., Žic, T., Vrbanec, D., et al. 2013, SoPh, 285, 295

Wang, Y.-M., \& Sheeley, N. R., Jr. 2002, ApJ, 567, 1211

Wang, Y.-M., Sheeley, N. R., Jr., Howard, R. A., St. Cyr, O. C., \& Simnett, G. M. 1999, Geophys. Res. Lett., 26, 1203

Wang, Y.-M., Sheeley, N. R., Jr., Walters, J. H., et al. 1998, ApJL, 498, L165

Webb, D. F., Howard, R. A., \& Jackson, B. V. 1996, in Solar Wind Eight, AIP Conf. Ser. 382, ed. D. Winterhalter, J. Gosling, S. Habbal, W. Kurth, \& M. Neugebauer (Melville, NY: AIP), 540

Weber, E. J., \& Davis, L., Jr. 1967, ApJ, 148, 217

Xu, F., \& Borovsky, J. E. 2015, J. Geophys. Res., 120, 70

Yang, L., He, J., Peter, H., et al. 2013, ApJ, 777, 16

Yokoyama, T., \& Shibata, K. 1996, PASJ, 48, 353

Zank, G. P., Nakanotani, M., Zhao, L.-L., Adhikari, L., \& Kasper, J. 2020, ApJ, 903, 1

Zhao, X. P., \& Hoeksema, J. T. 2010, SoPh, 266, 379 